\title{
Estudio de la influencia del método de moldeo de materiales cerámicos elaborados a partir de residuos en sus propiedades
}

\author{
M.T. COTES*, C. MARTÍNEZ, F.J. IGLESIAS, F.A.CORPAS \\ Departamento de Ingeniería Química, Ambiental y de los Materiales, Escuela Politécnica Superior de Linares. Universidad de Jaén. \\ *Corresponding author: Tef.:+34953648547-15; Fax:+34953648623 \\ e-mail: mtcotes@ujaen.es
}

\begin{abstract}
Las propiedades de los materiales cerámicos están íntimamente relacionadas con diversos factores entre los que destacan, el procedimiento de moldeo y la cinética de sinterización, puesto que condicionan la microestructura y propiedades de los materiales elaborados. En el presente trabajo se han formulado materiales a partir de arcillas comúnmente utilizadas en el área de Bailén (Jaén) y lodos procedentes de la depuración de aguas residuales urbanas. Se han moldeado los materiales mediante prensado y extrusión, por ser estas las técnicas más extendidas en la industria cerámica de la zona,con el fin de estudiar cuál de ellasse presenta como la más apropiada, estudiando propiedades de interés destacando la conductividad térmica y otras como: la resistencia a la compresión, la absorción de agua, la succión de agua, la densidad absoluta y aparenteentre otras.
\end{abstract}

Palabras clave: procesamiento, propiedades mecánicas, porosidad, conductividad.

A study of the influence of the method of shaping ceramic materials prepared with waste as one of their properties

The properties of ceramic materials are intimately related to a variety of factors, among them shaping procedure and sintering time. These factors condition the microstructure and properties of the materials developed. Our study has formed materials from clays commonly used in the area of Bailén (Jaén) and sludge proceeding from thewastewater treatment plant. We shaped the materials through pressing and extrusion, the techniques most widely used in the ceramics industry in this region. Our goal is to determine which technique is the most appropriate by studying properties of interest, highlighting the thermal conductivity, such as compressive strength, water absorption, water suction, absolute and apparent density, among others.

Keywords: processing, mechanical properties, porosity, conductivity.

\section{INTRODUCCIÓN}

El continuo crecimiento en la generación de residuos hace necesario no sólo aplicar medidas para reducir la generación de los mismos, sino también el reciclaje y reutilización a todos los niveles, tal y como se recoge en el Plan Nacional Integrado de Residuos 2007-2015 [1]. En concreto en España se producen anualmente 1.205.124 toneladas de lodos procedentes de instalaciones de depuración. De ellos, el 82,6 \% se empleó como fertilizante en la agricultura, el 7,9\% se depositó en vertedero y el 5,1\% fue incinerado con recuperación de energía. En el periodo 2000-2009 la generación de lodos se incrementó un $41,2 \%$, siendo la utilización agrícola el único destino que aumentó $(119,1 \%)$ [2]. En este sentido, la industria cerámica o la industria del cemento presentan procesos de fabricación que hacen especialmente viable la valorización de residuos $[3,4]$, bien aprovechando el poder calorífico procedente de la combustión de los mismos, bien incorporando el residuo a la estructura interna de los materiales formando parte de su propia matriz y pasando a ser un elemento inerte.[5-17]. También, cada vez son más demandados ladrillos con mayor capacidad de aislamiento térmico, una forma de obtenerlos es introducir en la matriz residuos orgánicos aligerantes [18-20]. De este modo el residuo deja de ser un problema para el medio ambiente e, incluso, en algunos casos, concede a los materiales fabricados con él prestaciones superiores a las del mismo material que no incorpora el residuo.

Enel casoespecífico delos materiales de cerámica estructural basados en arcilla cocida, se han realizado numerosos estudios con residuos de naturaleza orgánica e inorgánica. De un modo similar a la actuación de residuos orgánicos agrícolas, también se ha propuesto la incorporación de residuos urbanos y lodos de depuradora a las pastas cerámicas. Los residuos de naturaleza orgánica durante la sinterización de los materiales, sirven como combustible interno, dado su carácter orgánico, reduciendo la cantidad de combustible necesaria en el horno y dando lugar a productos más ligeros, permitiendo la fabricación de piezas de mayor tamaño. Además, las cenizas procedentes de la combustión pasan a formar parte de la matriz cerámica del producto, debido a la formación de fases cristalinas o vítreas durante el proceso de sinterización que ocurre con este tratamiento térmico. $[3,4,21]$. 
En este sentido, en el presente trabajo se han estudiado las propiedades tecnológicas de ladrillos fabricados por adición de diferentes cantidades lodos de depuración de aguas residuales urbanas a la matriz de arcilla. Se ha determinado la influencia del método de moldeoy la concentración óptima de residuo a adicionar para que se cumpla la normativa vigente aplicada a materiales de construcción estructurales.

\section{MATERIALES Y MÉTODOS.}

\subsection{Preparación de las materias primas}

Las materias primas utilizadas en este estudio son arcillas, que provienen de la industria cerámica de la ciudad de Bailén en la provincia de Jaén (España), lodos procedentes de la planta de tratamiento de aguas residuales urbanas de Linares en Jaén (España), así como el agua de la red de abastecimiento general.

La arcilla es recepcionada en el laboratorio y como tiene un tamaño de grano muy heterogéneo, se somete a un proceso de trituración en un molino de martillos. Posteriormente se pasa por un tamiz, en el caso del moldeo mediante prensado de 0,25 mm y de 0,5 mm para el moldeo mediante extrusión, para obtener un polvo fino y homogéneo. El control del tamaño de partícula es importante pues éste influye en las características físicas y mecánicas del producto final.

Los lodos de depuración de aguas residuales urbanas son recogidos en la planta de tratamiento y trasladados al laboratorio. Los residuos se secan en estufa a $90{ }^{\circ} \mathrm{C}$ durante $24 \mathrm{~h}$, para reducir su elevada humedad inicial y a continuación se someten a molturación en el molino citado, pasando seguidamente a un molino de bolas de ágata para luego tamizarlos y obtener un tamaño de grano de 0,25 mm para el prensado y $0,5 \mathrm{~mm}$ para el caso de la extrusión.

\section{2. Preparación de las muestras}

Con objeto de determinar el efecto de la adición de los lodos se utilizan diferentes cantidades entre el 0 y el $15 \%$ en peso. Después de pesar las cantidades adecuadas de residuo seco y arcilla se mezclan perfectamente en un mortero de porcelana, a continuación se procedió al moldeo de las muestras mediante prensa uniaxial de laboratorio Mega KCK-30 trabajando a 54,5 MPa (10 ton de presión de compactación), se añade en el proceso una cantidad de agua (10\% en peso), para obtener una adecuada plasticidad así como para evitar defectos en la etapa de compresión y que en el proceso de sinterización se produzcan porosidades y grietas. Utilizando idénticos porcentajes de lodo se conformaron piezas mediante unaextrusora de laboratorio de un husillo. La humedad requerida para este proceso fue del $22 \%$, ya que el moldeo por extrusión requiere una mayor plasticidad de la mezcla. Una vez conformadas, medidas y pesadas, se llevan a estufa a $110{ }^{\circ} \mathrm{C}$ durante $48 \mathrm{~h}$ para reducir su contenido en humedad. El secado es un fenómeno de superficie, el agua debe migrar a la superficie para su evaporación. A medida que avanza el secado, las partículas se van aproximando y la contracción aumenta [22]. La pérdida de agua no es lineal, pudiéndose distinguir tres etapas, en un primer momento se pierde el agua de los poros, en segundo lugar el agua residual y por último el agua absorbida.
Con objeto de que el material adquiera la resistencia y cohesión definitiva, se somete a la pieza secada a un proceso de cocción en horno que provoca el sinterizado de los granos, el aumento de densidad y variación de su porosidad, estos efectos generan una nueva contracción en el volumen de la pieza. En concreto, las muestras secas se llevan a un horno de laboratorio donde se calientan a una velocidad de $10{ }^{\circ} \mathrm{C} /$ min hasta llegar a $950{ }^{\circ} \mathrm{C}$, donde se mantienen $6 \mathrm{~h}$. Se utiliza esta temperatura por ser la utilizada con más frecuencia en la industria de la cerámica estructural. Una vez transcurrido ese tiempo las muestras se dejan enfriar mediante convección natural dentro del horno apagado.

\section{3. Ensayos realizados a las materias primas}

A las materias primas, se les han determinado características físicas y químicas de interés, detalladas a continuación.

Pérdida por calcinación a $950{ }^{\circ} \mathrm{C}$, se determinó por diferencia de pesada de la muestra sometida en horno de laboratorio a $950^{\circ} \mathrm{C}, 6 \mathrm{~h}$. Los resultados se calcularon en porcentaje.

La composición química de la arcilla, lodo fueron determinadas por fluorescencia de rayos $\mathbf{X}$ (FRX), en un equipo Philips Magix Pro (PW-2440). La composición elemental (contenido total de carbono, hidrógeno, nitrógeno y azufre) se determinó mediante combustión en atmósfera de oxígeno usando el analizador elemental CHNS-O Thermo Finnigan Elementary Analyzer Flash EA 1112.

El contenido en carbonatos se ha determinado mediante calcimetría de Bernard. El contenido en carbonatos es un factor que condiciona el proceso de producción del material y las propiedades de las piezas cocidas.

Para el análisis termogravimétrico (ATG) y termodiferencial (ATD) se ha empleado un equipo Mettler Toledo 850e donde las muestras, de entre 40 y $60 \mathrm{mg}$, fueron puestas en el crisol de platino y calentadas a una velocidad de y $10{ }^{\circ} \mathrm{C} / \mathrm{min}$ desde temperatura ambiente hasta $1000^{\circ} \mathrm{C}$. Los datos muestran los porcentajes de pérdida de peso en función de la temperatura y el diagrama ATD al mismo tiempo.

También se llevó a cabo un análisis dilatométrico de la arcilla y las mezclas utilizadas evaluándose así los cambios dimensionales, expansiones y contracciones que sufre el material por efecto de la temperatura y de las reacciones que ocurren en su interior, que generalmente llevan asociado un cambio de volumen del mismo. Para llevar a cabo el análisis dilatométrico de las muestras se empleó un Dilatómetro DIL 402 C.

Por último se determinó el poder calorífico superior de los lodos mediante una bomba calorimétrica Parr 1341 Plain, de acuerdo a la norma UNE 32 006:1995 [23].

\section{4. Ensayos realizados a los materiales cocidos}

Con objeto de determinar si los productos fabricados cumplen con la legislación vigente para su utilización como materiales de construcción, se han determinado sus propiedades físicas y mecánicas en los dos procesos de moldeo utilizados.

La pérdida de peso tras sinterización se obtiene pesando la pieza conformada después de la etapade cocción a $950^{\circ} \mathrm{C}$.

La densidad no es una propiedad térmica de los materiales pero se ha determinado porque los caracteriza disminuyendo o incrementando sus propiedades. Cuando en un material refractario se reduce la porosidad, existe un incremento de la 
TABLA 3.1. FRX DE ARCILLA Y CENIZAS DEL LODO.

\begin{tabular}{|c|c|c|c|c|c|c|c|c|c|c|c|c|}
\hline Material & $\mathrm{SiO}_{2}(\%)$ & $\underset{(\%)}{\mathrm{Al}_{2} \mathrm{O}_{3}}$ & $\begin{array}{c}\mathrm{Fe}_{2} \mathrm{O}_{3} \\
(\%)\end{array}$ & $\underset{(\%)}{\mathrm{MnO}}$ & $\underset{(\%)}{\mathrm{MgO}}$ & $\mathrm{CaO}(\%)$ & $\begin{array}{c}\mathrm{Na}_{2} \mathrm{O} \\
(\%)\end{array}$ & $\begin{array}{c}\mathrm{K}_{2} \mathrm{O} \\
(\%)\end{array}$ & $\underset{(\%)_{2}}{\mathrm{TiO}_{2}}$ & $\underset{(\%)}{\mathrm{P}_{2} \mathrm{O}_{5}}$ & $\underset{(\mathrm{ppm})}{\mathrm{ZrO}_{2}}$ & $\begin{array}{l}\text { LoI } \\
(\%)\end{array}$ \\
\hline Arcilla & 55.82 & 12.13 & 4.83 & 0.03 & 1.49 & 9.21 & 0.49 & 2.78 & 0.83 & 0.12 & 279.3 & 10.5 \\
\hline Cenizas Lodo & 46.37 & 20.33 & 8.55 & 0,28 & 2,19 & 11,15 & 0,36 & 3,25 & 0,85 & 5,89 & 161,7 & 0,05 \\
\hline
\end{tabular}

densidad. El aumento de porosidad, genera huecos en la matriz cerámica, parte de los cuales son ocupados por aire, estos poros actúan reduciendo la transmisión de calor por conducción en el interior de la pieza, y por tanto, la conductividad. [19, 24].

Los ensayos para la determinación de la densidad aparente seca se han llevado a cabo mediante la Norma UNE-EN 772-13 [25], empleando 10 probetas para cada residuo utilizado.

La absorción es una medida del porcentaje de volumen vacío o porosidad abierta de la pieza, es decir la porosidad constituida por una red continua de conductos capilares comunicados entre sí. Para ello se sumerge un ladrillo totalmente en agua durante un periodo prolongado. El ensayo se realizó siguiendo la norma UNE 67-027 [26].

La succión de un ladrillo es la cantidad de agua absorbida durante una inmersión parcial de corta duración y con este ensayo obtenemos una medida de la porosidad superficial interconectada de la pieza. Además esta propiedad está relacionada con la durabilidad del material. Para realizar este ensayo se sigue la norma UNE 67-031 [27].

La resistencia a lacompresión del ladrillo, es la carga unitaria aparente a la rotura, bajo un esfuerzo axial de compresión. Es una de las pruebas más importantes ya que la función del ladrillo es básicamente soportar esfuerzos de compresión en la construcción. Para este ensayo se tomaron series de quince probetas cocidas de cada muestra. El ensayo de resistencia a compresión se realizó siguiendo la norma UNE 67-026 [28] en una prensa de laboratorio.

También se ha llevado a cabo un ensayo de lixiviación para determinar la peligrosidad de los lixiviados de nuestras probetas, siguiendo la norma UNE-EN 12457-4 [29], esta norma es adecuada para determinar la movilidad tanto de compuestos orgánicos como inorgánicos presentes en líquidos, sólidos y residuos multifásicos.

Para la determinación experimental de la conductividad térmica se ha empleado el modelo de casa térmica,High insulation house de Phywe, para lo cual se dispone de un dispositivo al efecto, con paredes y techo aislados.

El estudio morfológico se ha llevado a cabo utilizando un microscopio electrónico de barrido JEOL modelo JSM5800, con sistema de microanálisis OXFORD modelo ISIS 486, previa preparación de las muestras en lámina plana y posterior metalización. Esta técnica utiliza electrones acelerados como fuente de radiación y la imagen se obtiene mediante barrido de un haz de electrones, procedentes de la fuente, que están enfocados sobre la superficie de la muestra. La resolución está entre 0,01 y $10 \mu \mathrm{m}$.

\section{RESULTADOS Y DISCUSIÓN}

\subsection{Materias primas}

Los datos de composición química y la pérdida por calcinación (LOI) de las cenizas de lodos de depuración de aguas residuales, así como de la arcilla utilizada para diseñar la composición de las muestras se presentan en la Tabla 3.1.

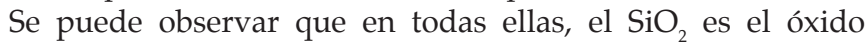
predominante.

La composición elemental de la arcilla y los lodos se muestra en la tabla 3.2.

TABLA 3.2. COMPOSICIÓN ELEMENTAL ARCILLA Y LODOS.

\begin{tabular}{|c|c|c|c|c|}
\hline Material & $\% \mathbf{C}$ & $\% \mathbf{H}$ & $\% \mathbf{N}$ & $\% \mathbf{S}$ \\
\hline Arcilla & $2.16 \pm 0.013$ & $0.37 \pm 0.001$ & $0.025 \pm 0.002$ & -- \\
\hline Lodos & $22.18 \pm 0.115$ & $3.18 \pm 0.026$ & $3.26 \pm 0.025$ & $1.23 \pm 0.338$ \\
\hline
\end{tabular}

El contenido de materia orgánica de la arcilla es pequeño, sin embargo el contenido de carbono, nitrógeno e hidrógeno del residuo es alto, de ahí el poder calorífico obtenido en la determinación mediante calorimetría. Estos resultados están de acuerdo con los obtenidos en el análisis termicogravimétrico de los lodos donde se aprecia una pérdida de masa total superior al $50 \%$.

Los carbonatos fueron determinados mediante calcimetría de Bernard. La mayoría de los carbonatos en la arcilla son cálcicos. La acción de los carbonatos es blanqueante, aumentan la absorción de agua y provocan un retraso en la fusión del mineral de arcilla. Por otra parte el $\mathrm{CO}_{2}$ formado durante la descomposición del carbonato cálcico, se acumula en los poros de la pieza y los dilata contribuyendo a neutralizar las contracciones durante la cocción y a aumentar la porosidad del producto cocido. En consecuencia si se quieren obtener probetas porosas y ligeras entonces el contenido en carbonato podrá ser alto [30]. Las determinaciones realizadas sobre 15 muestras de nuestra arcilla muestran un contenido medio de $8,53 \pm 0.07 \%$, de $\mathrm{CaCO}_{3}$.

El ATG-ATD de la arcilla y de los residuos empleados hasta $900{ }^{\circ} \mathrm{C}$ se muestra en la Figura 3.1. En el caso de la arcilla (Figura 3.1.a), desde temperatura ambiente hasta $100{ }^{\circ} \mathrm{C}$, se observa un pico endotérmico a $60^{\circ} \mathrm{C}$, asociado a la pérdida de humedad, con una pérdida de peso del 1,4\%. Entre los 250 ${ }^{\circ} \mathrm{C}$ y $500{ }^{\circ} \mathrm{C}$ la pérdida de peso del $0,8 \%$ se puede atribuir a la combustión de materia orgánica que está asociada a un proceso exotérmico. Entre 450 y $630^{\circ} \mathrm{C}$, se lleva a cabo la deshidroxilación de los minerales de la arcilla, como la descomposición de la caolinita y el agua estructural de la illita, mostrando la curva de ATD un pico endotérmico centrado a $565^{\circ} \mathrm{C}$. A partir de $600{ }^{\circ} \mathrm{C}$, se aprecia la descomposición del carbonato de calcio con la liberación de $\mathrm{CO}_{2}$, que se asocia con el proceso endotérmico centrado a $780{ }^{\circ} \mathrm{C}$, siendo en esta fase la pérdida de peso del 9,5\%.

La figura $3.1 b$ muestra las curvas de ATG-ATD de los lodos procedentes dela depuración de aguas urbanas. Desde temperatura ambiente hasta $200{ }^{\circ} \mathrm{C}$ se observa una pérdida 
de peso del 5,8\%, producida por la liberación de agua físicamente adsorbida, observándose un pico endotérmico a aproximadamente $100{ }^{\circ} \mathrm{C}$. La descomposición de la materia orgánica se produce entre los 200 y los $550{ }^{\circ} \mathrm{C}$, con una pérdida de peso de $37,5 \%$. El primer pico exotérmico entre 200 y $400^{\circ}$ $\mathrm{C}$ se asocia a la descomposición de materiales biodegradables, la materia orgánica digerida y bacterias muertas, junto con las emisiones de compuestos semivolátiles [31, 32, 33] mientras que el segundo pico exotérmico entre 400 y $550{ }^{\circ} \mathrm{C}$ se atribuye a la oxidación de otros materiales oxidables en la muestra. Finalmente, el último pico endotérmico, a $700{ }^{\circ} \mathrm{C}$ puede ser debido a la descomposición del carbonato de calcio (calcita), con liberación de $\mathrm{CO}_{2}$, una pérdida de peso del 9,8\%.
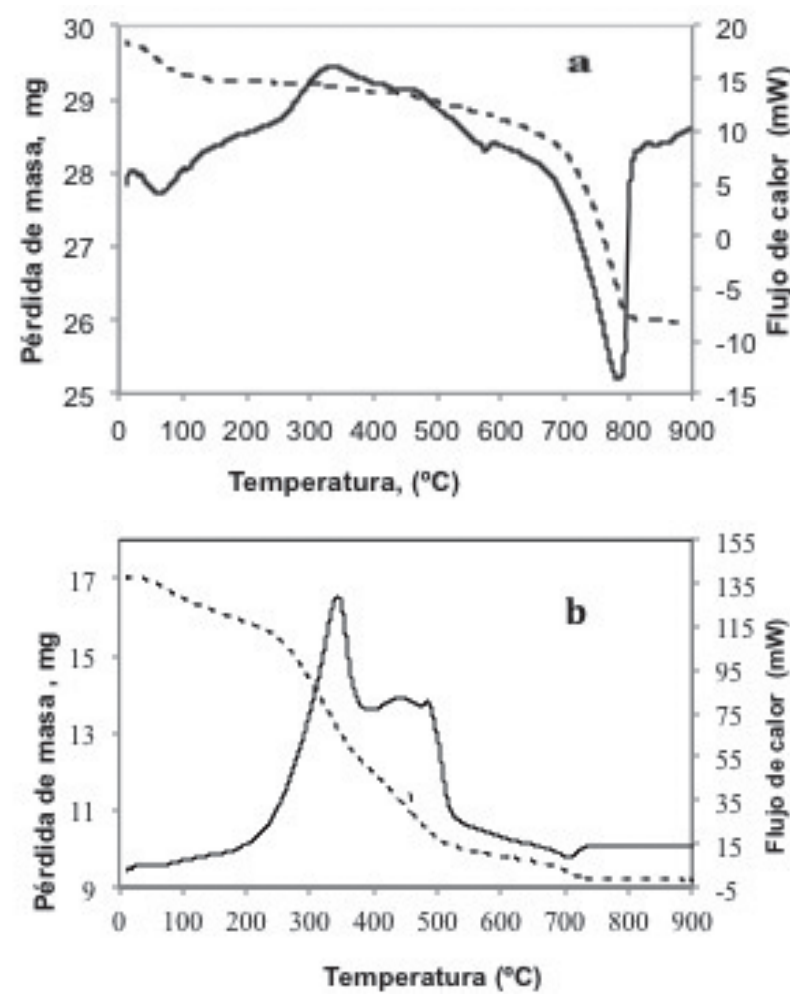

Figura 3. 1. ATG (-----) -ATD ( ) a) Arcilla; b) Lodos EDAR urbana.

En la Figura 3.2.a, se muestra la curva dilatométrica de la arcilla hasta $1100{ }^{\circ} \mathrm{C}$. En ella se observa durante el calentamiento una dilatación inicial con una pendiente constante hasta aproximadamente $400{ }^{\circ} \mathrm{C}$. La inflexión observada es debido al secado por la eliminación de la humedad. Posteriormente dicha dilatación se produce a una mayor velocidad entre 500 y $600{ }^{\circ} \mathrm{C}$ relacionada con el cambio alotrópico de cuarzo- $\alpha$ a cuarzo- $\beta$. Esta transformación va acompañada de un cambio de volumen del $3 \%$. La dilatación prosigue hasta que, alrededor de $730{ }^{\circ} \mathrm{C}$ y se detiene. Desde esta temperatura hasta $942{ }^{\circ} \mathrm{C}$ se observa una contracción debido a la descomposición de carbonato cálcico en $\mathrm{CO}_{2}$ y $\mathrm{CaO}$ y a la formación de fase líquida que con la temperatura va disminuyendo su viscosidad, dando lugar al comienzo de la sinterización de las partículas de mineral arcilloso, produciendo una disminución de la superficie específica. La eliminación del gas favorece en el material una estructura porosa, mientras que la presencia del $\mathrm{CaO}$ da lugar a nuevas reacciones en la arcilla a temperaturas superiores. Entre los 700 y $1000{ }^{\circ} \mathrm{C}$ tienen lugar varias reacciones entre la $\mathrm{SiO}_{2}$ y la $\mathrm{Al}_{2} \mathrm{O}_{3}$ procedentes de la descomposición de los minerales de arcilla y los cationes presentes en la arcilla $(\mathrm{Mg}, \mathrm{Ca}, \mathrm{Fe})$ que explican el aumento de volumen. Dado el contenido de carbonato cálcico, es el óxido cálcico el que reacciona dando lugar a la formación de anortita: $\left(\mathrm{CaO} . \mathrm{Al}_{2} \mathrm{O} 32 \mathrm{SiO}_{2}\right)$, wollastonita $\left(\mathrm{CaO} . \mathrm{SiO}_{2}\right)$ y gehlenita $\left(2 \mathrm{CaO} \cdot \mathrm{Al}_{2} \mathrm{O}_{3} \cdot \mathrm{SiO}_{2}\right)$ [34].

La cristalización de estas nuevas fases cálcicas ocurre en general en partículas de pequeño tamaño e impide el desarrollo de una cantidad de fase amorfa significativa, confiriendo a las piezas obtenidas una porosidad elevada. Dichas fases se mantienen estables hasta temperaturas del orden de $1100{ }^{\circ} \mathrm{C}$. La fuerte contracción que se observa es debida a la formación de abundante fase líquida. Se observa que la temperatura óptima se situaría en torno a los $900-950{ }^{\circ} \mathrm{C}$.

$\mathrm{Al}$ adicionar lodo el comportamiento será muy similar a la muestra de arcilla, se producirá una disminuyendo de los picos de temperaturadebido a la aportación de carbonatos procedentes de los lodos, esto contribuirá a disminuir el consumo energético en la cocción de las piezas, [35, 36]. En estas condiciones hay que tener en cuenta los cambios que introduce la presencia de carbonatos en las propiedades mecánicas de las probetas, que consiste básicamente en reducción de resistencia y durabilidad, esta aportación según distintos autores no debe superar el $10 \%[37,38]$.

La acción fundente de los carbonatos durante el tratamiento térmico está asociada a la reducción de la temperatura de sinterización, ya que estos minerales influyen en lastransformaciones mineralógicas que tienen lugar en los minerales de arcilla durante su cocción y permite la formación de mayor cantidad de fases vítreas a menor temperatura comparado con materiales que no contengan carbonatos [35, $36,39]$.

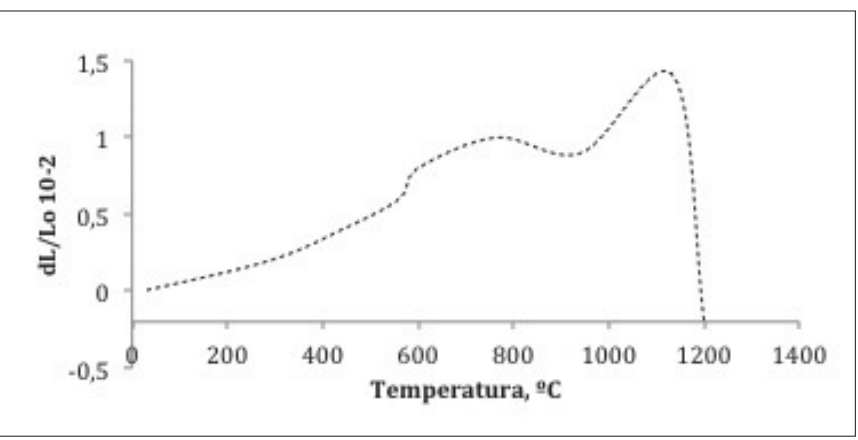

Figura 3.2. Curva dilatométrica de la arcilla.

Por último se determinó el poder calorífico de los lodos en una bomba calorimétrica PARR 1341 obteniéndose los siguientes resultados:

TABLA 3.3. PODER CALORÍFICO SUPERIOR E INFERIOR LODOS DEPURADORA CAL/G.

\begin{tabular}{|l|c|}
\hline $\begin{array}{l}\text { Poder calorífico superior (PCS, cal/g) } \\
\text { Lodo urbano }\end{array}$ & $1235,40 \pm 36,14$ \\
\hline $\begin{array}{l}\text { Poder calorífico inferior }(\mathbf{P C I}, \mathbf{c a l} / \mathbf{g}) \\
\text { Lodo urbano }\end{array}$ & $1067,97 \pm 36,14$ \\
\hline
\end{tabular}

Los resultados muestran que agregar este tipo de residuos para el porcentaje máximo estudiado (15\% en peso) proporcionaráun ahorro del combustible necesario en la elaboración de las piezas. Estos resultados están de acuerdo 
con los obtenidos en la TGA-DTA y el análisis elemental, que muestran una pérdida de masa de los lodos debido fundamentalmente al contenido orgánico de los mismos.

\subsection{Materiales moldeos}

Se da en la tabla 3.4 la contracción (expresada en \% peso) en función del proceso de moldeado, acompañada de su desviación estándar.

TABLA 3.4. CONTRACCIÓN (\% PESO) ENFUNCIÓN DEL MÉTODO DE MOLDEO Y DESPUÉS DEL TRATAMIENTO A950 ${ }^{\circ} \mathrm{C} / 24+6$ HORAS

\begin{tabular}{|c|c|c|}
\hline $\begin{array}{c}\text { Porcentaje lodo, } \\
\% \text { peso }\end{array}$ & $\begin{array}{c}\text { Pérdida de peso, } \\
\text { compresión }\end{array}$ & $\begin{array}{c}\text { Pérdida de peso, } \\
\text { extrusión }\end{array}$ \\
\hline 0 & $0.105 \pm 0.005$ & $0.107 \pm 0,015$ \\
\hline 2 & $0.147 \pm 0.003$ & $0.118 \pm 0,014$ \\
\hline 4 & $0.151 \pm 0.003$ & $0.116 \pm 0,093$ \\
\hline 6 & $0.157 \pm 0.001$ & $0.116 \pm 0,081$ \\
\hline 8 & $0.132 \pm 0.001$ & $0.113 \pm 0,045$ \\
\hline 15 & $0.180 \pm 0.002$ & $0.167 \pm 0,021$ \\
\hline
\end{tabular}

En ambos casos se observa que la pérdida de peso aunque aumenta no lo hace de manera apreciable hasta porcentajes elevados de adición de lodos.

La densidad absoluta seca $(\mathrm{Pn}, \mathrm{u})$ y aparente seca $(\mathrm{Pg}, \mathrm{u})$ se determinó mediante las normas UNE-EN 772-13 y UNE-EN 772-3. Los resultados obtenidos se muestran el la tabla 3.5.

Tanto en las muestras obtenidas por prensado como por extrusión se aprecia una disminución de los valores de densidad absoluta y aparente seca, debido a la creciente porosidad creada por la adición mayores porcentajes de lodo. Esta disminución es más acusada para el caso del prensado, un $17 \%$ para la densidad absoluta seca y un $15 \%$ para la aparente seca. En el caso de las muestras obtenidas por extrusión los valores descienden un $3 \%$ para la densidad absoluta seca y un $8 \%$ para la aparente seca. Esto se confirma con los valores obtenidos para la absorción de agua que se incrementan de forma proporcional a las cantidades de lodo adicionadas.

Para el caso de la absorción de agua los resultados se muestran en la figura 3.3.

En ambos casos se incrementan los valores de absorción entre un 50 para el caso de la extrusión y un $60 \%$ para el prensado en comparación con las probetas elaboradas sólo con arcilla. Esta propiedad está relacionada con la porosidad abierta generada en el interior del material, debido

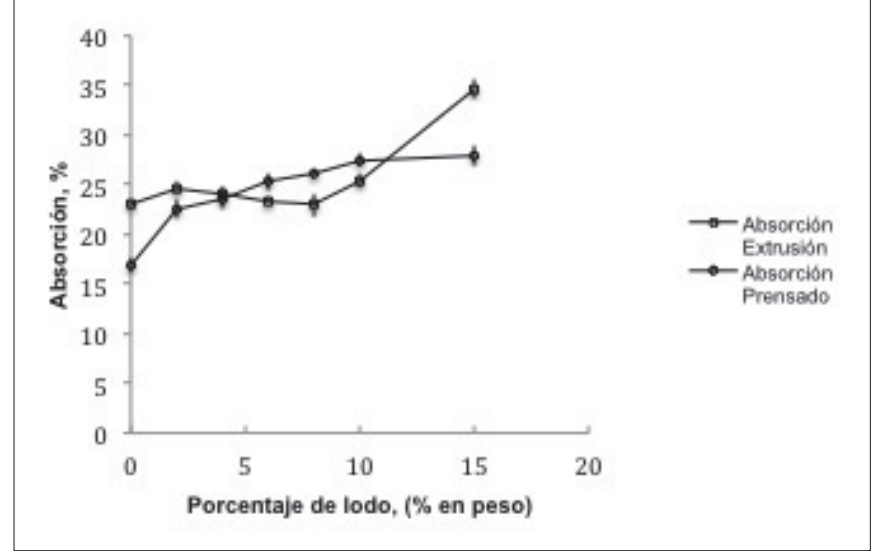

Figura 3.3. Absorción de agua frente a porcentaje de lodo adicionado (\% en peso).

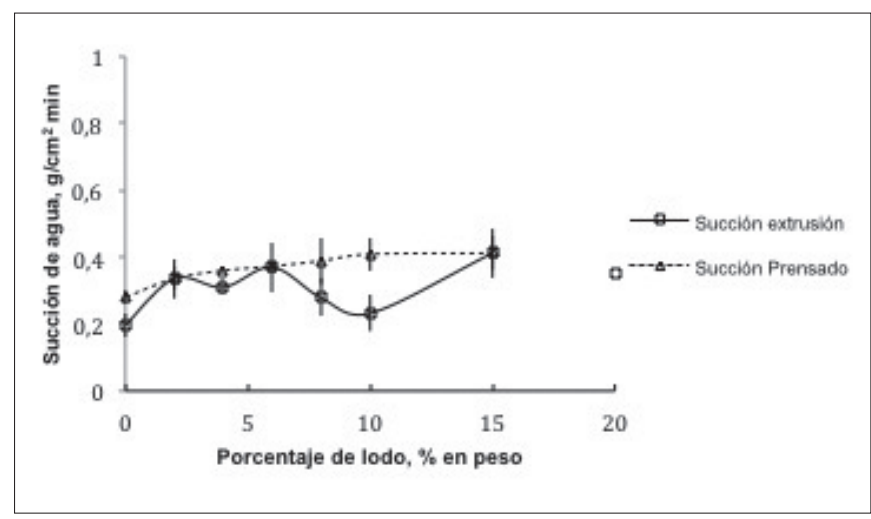

Figura 3.4. Succión de agua frente a porcentaje de lodo adicionado (\% en peso).

a la combustión de la materia orgánica presente en el residuo adicionado.

La succión se presenta en la gráfica 3.4, Se puede observar que hay un aumento de la succión de agua tras la sinterización y un ligero aumento cuando el porcentaje de lodos añadidos es mayor, resultado esperado pues al someter a las piezas a estas temperaturas provoca el aumento de la porosidad superficial interconectada y una clara tendencia al aumento en la succión de agua tanto en el moldeo por prensado como por extrusión. Siendo menos acentuado para porcentajes del 0 al $10 \%$ por extrusión. Esta propiedad afecta y mucho a la calidad del material final y a su durabilidad por lo que altos contenidos en lodos nos podrían provocar piezas con defectos y una clara tendencia a la succión de agua y por tanto una menor durabilidad.

TAbla 3.5. Densidad absoluta SECA y APARENTE SECA $\left(\mathrm{KG} / \mathrm{M}^{3}\right)$

\begin{tabular}{|c|c|c|c|c|}
\hline$\%$ en peso lodos & $\begin{array}{l}\text { Pn, u, kg/m } \mathrm{m}^{3} \\
\text { Prensado }\end{array}$ & $\begin{array}{l}\mathrm{Pg}, \mathrm{u}, \mathrm{kg} / \mathrm{m}^{3} \\
\text { Prensado }\end{array}$ & $\begin{array}{l}\text { Pn, u, kg/m } \mathrm{m}^{3} \\
\text { Extrusión }\end{array}$ & $\begin{array}{l}\mathrm{Pg}, \mathrm{u}, \mathrm{kg} / \mathrm{m}^{3} \\
\text { Extrusión }\end{array}$ \\
\hline $0 \%$ & $1615,13 \pm 1,09$ & $1586,57 \pm 0,02$ & $1525,23 \pm 24,24$ & $1333,02 \pm 32,73$ \\
\hline $2 \%$ & $1616,22 \pm 4,56$ & $1566,11 \pm 0,06$ & $1522,07 \pm 25,74$ & $1312,45 \pm 33,95$ \\
\hline $4 \%$ & $1546,34 \pm 3,22$ & $1474,98 \pm 0,02$ & $1510,09 \pm 30,10$ & $1447,22 \pm 487,55$ \\
\hline $6 \%$ & $1423,88 \pm 8,51$ & - & $1486,02 \pm 13,27$ & $1452,75 \pm 92,27$ \\
\hline $8 \%$ & $1420,19 \pm 8,66$ & $1409,29 \pm 0,03$ & $1507,98 \pm 86,28$ & $1268,58 \pm 173,75$ \\
\hline $10 \%$ & $1409,75 \pm 21,30$ & $1381,06 \pm 0,02$ & $1477,37 \pm 115,95$ & $1253,35 \pm 92,29$ \\
\hline $15 \%$ & $1340,39 \pm 2,13$ & $1348,09 \pm 0,01$ & $1466,21 \pm 49,98$ & $1223,38 \pm 93,19$ \\
\hline
\end{tabular}


Referente a las propiedades mecánicas, tan importantes en estos materiales, se ha llevado a cabo ensayos de compresión de acuerdo a la norma UNE, obteniéndose los resultados mostrados en la Figura 3.5, como se puede observar hay una disminución no muy acusada de la resistencia de las piezas, para el caso de utilizar únicamente arcilla, la pieza resiste hasta presiones de más de $30 \mathrm{Mpa}$, sin embargo para las probetas mixtas esta resistencia cae por debajo de este valor se observa una clara tendencia a la baja con el aumento del porcentaje de lodos añadidos, lo que hace suponer que hay un aumento de porosidad y por tanto una disminución de resistencia mecánica, lo que queda confirmado por los valores anteriormente obtenidos de densidad y absorción de las probetas elaboradas. Esa disminución es más acusada para las piezas obtenidas mediante extrusión, debido probablemente a la presión aplicada en el proceso de moldeo. Se aprecia también que para porcentajes de lodo elevados, a partir del $8 \%$ y en extrusión, no se cumpliría con la normativa vigente para materiales de uso estructural $\left(10 \mathrm{~N} / \mathrm{mm}^{2}\right)$.

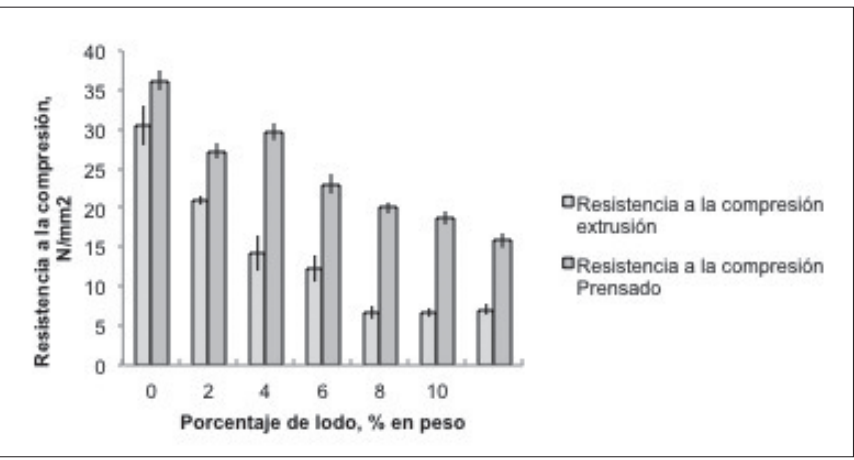

Figura 3.5. Resistencia a la compresión (MPa) frente a porcentaje en peso de lodo adicionado.
Los resultados obtenidos en el test de lixiviación indican que con la legislación actual no se podrían clasificar como "peligrosos" los lixiviados de nuestras probetas, ya que ninguna de las concentraciones de los elementos determinados supera las que indica la norma. En la Tabla 3.6 se muestran los resultados del test de lixiviación efectuado y los límites legales permitidos para esos elementos.

Los resultados de la determinación de la conductividad térmica. Se muestran en la Tabla 3.7 en función del contenido lodos. Se observa que a medida que se introducen mayores porcentajes la conductividad térmica disminuye, debido probablemente a la formación de porosidad cerrada debido a la combustión de la fracción orgánica del residuo. La porosidad cerrada es la causa de la capacidad de aislamiento térmico de los materiales.

Así los poros grandes originarán un aumento de la conductividad térmica, los más finos y uniformes le dan mayor capacidad de aislamiento térmico [40]. Por el contrario la ausencia de porosidad supone un incremento de densidad $\mathrm{y}$, casi siempre, de conductividad térmica [41, 42].

Los valores de la conductividad también están relacionados, como se ha mencionado, con la absorción de agua y el tipo de porosidad. Así a mayor contenido en lodos, mayor porosidad, y por tanto menores valores de conductividad térmica. La relación se puede observar en la Figura 3.6, donde se representan ambos valores frente al contenido en lodo.

Se observa que a partir del $2 \%$ se produce una disminución de los valores de la conductividad, ello podría ser debido a que al aumentar el porcentaje de lodos se produce un incremento de porosidad cerrada lo que hace que k disminuya apreciablemente. A porcentajes mayores se originará un incremento de porosidad abierta, lo que está en consonancia con los resultados obtenidos en las determinaciones de densidad, absorción de agua y la disminución de la resistencia a la compresión.

TABLA 3.6. RESUlTADOS TEST DE LIXIVIACIÓN SEGÚN EL PORCENTAJE EN PESO DE LODO AÑADIDO Y LÍMITE LEGAL ADMITIDO (UNE-EN 12457-4)

\begin{tabular}{|c|c|c|c|c|c|c|c|}
\hline $\begin{array}{c}\text { Muestra } \\
\text { Elemento }\end{array}$ & M1 & M2,5 & M5 & M7,5 & M10 & M15 & $\begin{array}{c}\text { ppb máximas permitidas } \\
\text { Test TCLP }\end{array}$ \\
\hline Cr & 924,1 & 882,9 & 706,6 & 809,5 & 478,8 & 341,3 & 5000 \\
\hline Ni & 2,17 & 10,74 & 4,03 & 2,11 & 25 & 1,65 & $2000-400$ \\
\hline $\mathbf{C u}$ & 17,67 & 29,75 & 18,8 & 23,8 & 39,27 & 29,32 & $10000-2000$ \\
\hline Zn & 1,19 & 5,05 & 86,15 & 1,17 & 84,59 & 15,92 & $10000-2000$ \\
\hline As & 26,22 & 33,48 & 78,62 & 32,23 & 137,4 & 5,47 & $1000-200$ \\
\hline Se & 1,72 & 9,47 & 1,46 & 1,52 & 14,44 & 4,71 & 1000 \\
\hline Ag & 0,81 & 5,22 & 0,54 & 0,66 & 4,84 & 2,4 & 5000 \\
\hline $\mathbf{C d}$ & 0,53 & 6,42 & 0,07 & 0,08 & 6,78 & 0,15 & $500-100$ \\
\hline Pb & 1,87 & 11,16 & 0,66 & 0,33 & 13,12 & 0,66 & $2000-400$ \\
\hline
\end{tabular}

TABLA 3.7. VALORES DE CONDUCTIVIDAD TÉRMiCA DE LAS PROBETAS

\begin{tabular}{|c|c|c|c|c|c|c|c|}
\hline $\begin{array}{c}\text { Porcentaje lodo } \\
\text { adicionado, en peso }\end{array}$ & $0 \%$ & $2 \%$ & $4 \%$ & $6 \%$ & $8 \%$ & $10 \%$ & $15 \%$ \\
\hline $\begin{array}{c}\text { Conductividad W/mK, } \\
\text { compresión }\end{array}$ & $\begin{array}{c}0,183 \\
\pm 0,014\end{array}$ & $\begin{array}{c}0,197 \\
\pm 0,016\end{array}$ & $\begin{array}{c}0,163 \\
\pm 0,012\end{array}$ & $\begin{array}{c}0,150 \\
\pm 0,008\end{array}$ & $\begin{array}{c}0,160 \\
\pm 0,013\end{array}$ & $\begin{array}{c}0,160 \\
\pm 0,016\end{array}$ & $\begin{array}{c}0,128 \\
\pm 0,011\end{array}$ \\
\hline Descenso, \% & - & $-7,65$ & 10,92 & 18,03 & 12,57 & 12,57 & 30,05 \\
\hline $\begin{array}{c}\text { Conductividad W/mK, } \\
\text { extrusión }\end{array}$ & $\begin{array}{c}0,190 \\
\pm 0,012\end{array}$ & $\begin{array}{c}0,146 \\
\pm 0,012\end{array}$ & $\begin{array}{c}0,165 \\
\pm 0,019\end{array}$ & $\begin{array}{c}0,133 \\
\pm 0,013\end{array}$ & $\begin{array}{c}0,143 \\
\pm 0,010\end{array}$ & $\begin{array}{c}0,145 \\
\pm 0,029\end{array}$ & $\begin{array}{c}0,142 \\
\pm 0,006\end{array}$ \\
\hline Descenso, \% & - & 23,15 & 13,15 & 30 & 24,74 & 23,68 & 25,26 \\
\hline
\end{tabular}




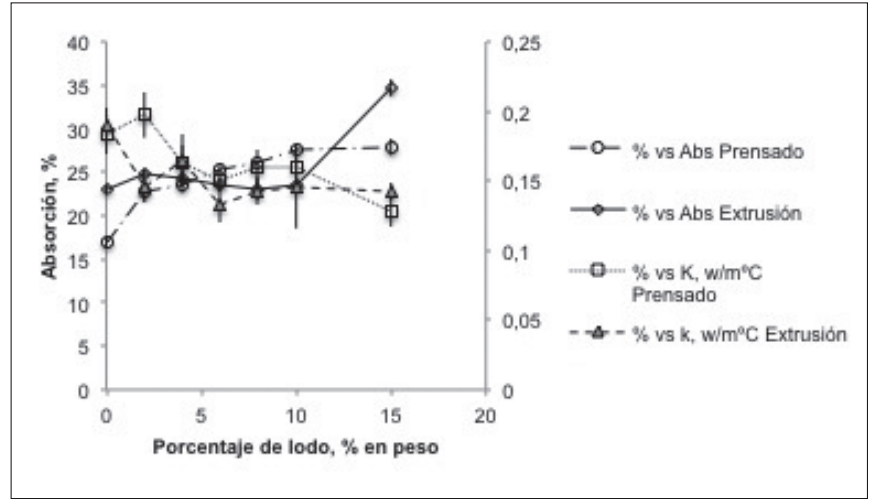

Fig. 3.6. Curva de variación de la absorción y conductividad térmica de piezas incorporando diferentes porcentajes en peso de lodo de depuradora.

Para la muestra del $15 \%$, la presencia de materia orgánica será mayor por tanto en el proceso de combustión dará lugar al desarrollo de mayor porosidad en el interior del material, cerrada y abierta, dado el descenso de la conductividad térmica.

En cuanto al estudio morfológico, se presentan las micrografías correspondientes a las muestras de arcilla y del 5 y $15 \%$ en peso de lodos adicionados. Se puede apreciar que los macroporos originados por la calcinación de los lodos presentan una forma redondeada, con bordes bien definidos. Esto se relaciona con los resultados obtenidos de la conductividad térmica, se produce una disminución de la conductividad, por tanto una mayor capacidad de aislamiento térmico debido a los poros llenos de aire estanco incorporados dentro de su masa.

La cantidad y calidad de la porosidad cerrada comporta determinadas propiedades que vienen, de hecho relacionadas entre ellas, como es la densidad y la conductividad térmica.La ausencia de porosidad supone un incremento de densidad y, casi siempre, de conductividad térmica.[22]

\section{CONCLUSIONES.}

En principio se concluye que la adición de lodo procedente de una planta de tratamiento de aguas residuales urbanas genera un aumento de la porosidad en la matriz cerámica, siendo ésta mayoritariamente cerrada a porcentajes menores y aumentando la fracción abierta para las muestras con un mayor contenido en lodo. También se puede concluir que porcentajes mayores del $10 \%$, no serían recomendables puesto que afectan a las propiedades mecánicas de las probetas elaboradas y además presentan desde su inicio defectos morfológicos.

Aunque se produce un descenso de la resistencia a la compresión, como era previsible, por el aumento de la porosidad en las piezas. Todos los porcentajes de lodo cumplen con la exigencia de la norma en el caso de moldeo por presión y hasta porcentajes de lodo del $8 \%$ en caso de extrusión.

Todas las probetas elaboradas cumplen la legislación en materia de valorización de residuos (Test de lixiviación).

Se produce una mejora de las propiedades térmicas del material, aumenta su capacidad de aislamiento térmico.

En cuanto al proceso de moldeo se concluye que la extrusión es el procedimiento más adecuado, debido a su mayor versatilidad, así como a su capacidad de producción más elevada, al trabajar en continuo,aspecto éste muy beneficioso a nivel industrial.Esto implica una mayor facilidad de operación respecto al método de conformado por prensado que trabajaría en semicontinuo. También se concluye que en las propiedades estudiadas no hay diferencias apreciables entre los dos métodos de conformado empleados, siendo por tanto preferible la extrusión por las ventajas antes indicadas.

Por último el probeta obtenida con un $6 \%$ de lodo tanto en compresión como en extrusión es la que presenta mejores características aislantes y a su vez cumple con la normativa de resistencia a la compresión.

\section{AGRADECIMIENTOS}

Los autores del trabajo agradecen la colaboración de Arcilllas Bailén S.L. y la Estación depuradora de aguas residuales urbanas de la ciudad de Linares, por el suministro de las arcillas y lodos respectivamente y a los Servicios Técnicos de Investigación de la Universidad de Jaén por el apoyo prestado en algunas de las determinaciones incluidas en este trabajo.

\section{REFERENCIAS}

1. Spanish Sustainable Development Strategy, 2007

2. Eurostat 2009. http:/ / epp.eurostat.ec.europa.eu/.

3. Ravaglioli A., Fiori C., Fabbri B.: "Materie prime ceramiche. Argille, materiali non argillosi e sottoprodotti industriali". Faenza Editrice S.P.A. Faenza. (1989).

4. Sánchez-Muñoz L., Carda Castelló J.B.: “Materiales residuales”. En pp. 159-160 de Materias primas y aditivos cerámicos. Ed. Faenza Editrice Iberica, S.L. Castellón. (2002).
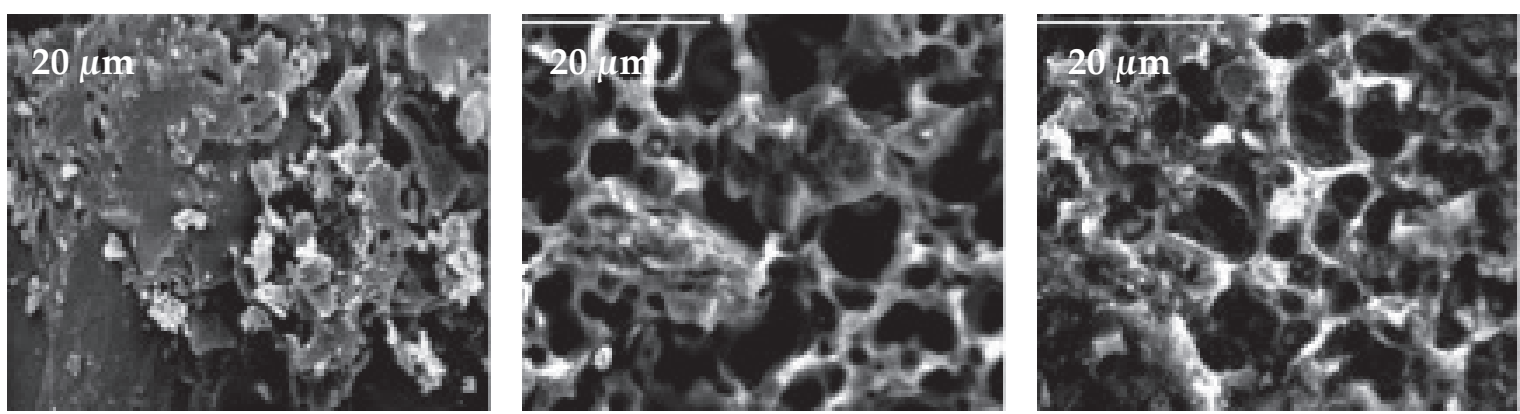

Fig. 3.7.Fotografías microscopio electrónico (SEM)

a)Probetas Arcilla, muestras, b) $5 \%$ y c) $15 \%$, en peso de lodo. 
5. Romero M., Rincón J. Ma.: "Procesos de vitrificación/cristalización controlada aplicados al reciclado de residuos inorgánicos industriales", Bol. Soc. Esp. Ceram. Vidrio.Vol. 39 , nº1, (2000), pp. 155-163.

6. Vieira C.M.F., Soares T.M., Sánchez R., Monteiro S.N.: "Incorporation of granite waste in red ceramics". Mater. Sci. and Eng. Vol. 373, 2004, pp. 115121.

7. Andreola F., Barbieri L., Corradi A., Lancelloti.:“CRT glass state of the art. A case study: Recycling in ceramic glazes“. J. Eur. Ceram. Soc. Vol. 27n $2-3$ (2007), pp.1623-1630.

8. Monteiro S.N., Vieira C.M.F.: "Effect of oily waste addition to clay ceramic". Ceram. Inter. Vol.31, (2005), pp. 353-358.

9. Naga S.M., El-Maghraby A.:"Industrial wastes as raw materials for tile making". Key Eng. Mat. Vol. 206, (2002), pp. 1787-1790.

10. Barbieri L., Bonomartini A.C., Lancelotti I.:"Alkaline and alkaline-earth silicate glasses and glass-ceramics from municipal and industrial wastes". J. Eur. Ceram. Soc.Vol. 20,nº 14-15, (2000), pp. 2477-2483.

11. Pelino M.: "Recycling of zinc-hydrometallurgy wastes in glass and glass ceramics materials". Waste Manag. Vol.20, pp., nº 7, (1998) 561-568.

12. Marabini A.M., Plescia P., Maccari D., Burragato F., Pelino M.:"New materials from industrial and mining wastes: glass-ceramics and glass and rock-wool fibre". Int. Jou.r Mineral Process. Vol.53, n 1-2, (1998), pp.121134.

13. Queralt I., Querol X., Lopez Soler A., Plana F.:“Use of coal fly ash for ceramics: A case study for a large Spanish power station". Fuel. Vol.76, n 8, (1997), pp. 787-791.

14. Karamanov A., Taglieri G., Pelino M.:"Iron-rich sintered glass-ceramics from industrial wastes" J. Am. Ceram. Soc. Vol. 82, n 11, (1999), pp. 30123016.

15. Pontikes Y., Nikolopoulos P., Angelopoulos G.N.:"Thermal behaviour of clay mixtures with bauxite residue for the production of heavy-clay ceramics", J. Eur. Ceram. Soc. Vol. 27, n 2-3, (2007), pp. 1645-1650.

16. Gorokhovsky A.V.: "Inorganic wastes in manufacturing of glass-ceramics: slurry of phosphorous fertilizaer production and oil shale ash". Materials Lett. Vol.51, n 3, (2001), pp. 281-284.

17. Gualtieri A.F., Taratglia A.:"Thermal decomposition of asbestos and recycling in traditional ceramics". J. Eur. Ceram. Soc.Vol. 20, n 9, (2000), pp.1409-1418

18. Couto D.M., Ringuedé A., Silva R.F.,Labrincha J.A., Rodrigues C.M.S. "Metallurgical Sludge in Clay-Based Fired Materials". Amer. Ceram. Bull. Vol. 82, n 12, (2003), pp. 9101-9103.

19. I. Demir.: "Effect of organic residues addition on the technological properties of clay bricks". Waste Manag.Vol. 28, (2008), pp. 622-627.

20. Martínez C., Cotes M.T., Corpas F.A.: "Recovering wastes from the paper industry: Development of ceramic materials". Fuel Process. Tech. http:// dx.doi.org/10.1016/j.fuproc.2011.10.017.

21. Chakraverty A., Mishra P., Banerjee H.D.: "Investigation of combustion of raw and acid-leached rice husk for production of pure amorphous silica". J. Mater. Sci. Vol. 23 nº 1, (1988), pp. 21-24.

22. Elías, X.:Optimización de los Procesos Cerámicos Industriales, ponencias ID 57, 58, 59 y 60. http :/ / www .cnpml .org/ html/ archivos/Ponencias. (2001).

23. Norma UNE 32-006 (1995). Combustibles minerales sólidos. Poder calorífico mediante determinación en calorímetro automático.

24. G. Tufiño,LL. Vieira,V. Gurrero.: Propiedades térmicas de arcillas para ser utilizadas como material refractario. Centro de desarrollo tecnológico sustentable. Escuela Politécnica Nacional de Quito. Ecuador. http:/ / www. Cdts.espol.edu.ec/principal.htm. (2007)

25. UNE-EN 772-13, Métodos de ensayo de piezas para fábrica de albañilería. Determinación de la densidad absoluta seca y de la densidad aparente seca de piezas para fábrica de albañilería. (excepto piedra natural), (2001).

26. UNE 67-027, Ladrillos. De arcilla cocida. Determinación de la Absorción de agua, (1984).

27. UNE 67-031, Ladrillos de arcilla cocida. Determinación de la Succión de agua, (1985)

28. UNE 67-026. Ladrillos de arcilla cocida. Determinación de la Resistencia a la compresión. (1986).

29. UNE-EN 12457-4. Caracterización de residuos. Lixiviación. Ensayo de conformidad para la lixiviación de residuos granulares y lodos. Parte 4 Ensayo por lotes de una etapa con una relación líquido-sólido de 10 1/ kg para materiales con un tamaño de partícula inferior a $10 \mathrm{~mm}$ (con o sin reducción de tamaño). (2003)

30. F. Fernández Abajo.:Manual sobre fabricación de Baldosas, Tejas y Ladrillos. Terrassa. Beralmar S.A. (2000)

31. Conesa, J.:Basiccourse in thermal analysis. In: Thermogravimetry, Kinetics of Reactions and Differential Thermal Analysis. Club Univ. (2000)

32. Font, R., Fullana, A., Conesa, J.A., Llavador, F.:"Analysis of pyrolysis and combustion of different sewage sludges by TG". J. Anal. App. Pyrolysis. Vol. $58-592, n^{\circ} 2$ (2001) , pp.927-941.

33. Calvo, L.F., Otero, M., Jenkins, B.M., García, A.I.: Morás, A. “Heating process characteristics and kinetics of sewage sludge in different atmospheres". Ther- mochim. Acta. Vol. 409, n 2, (2004), pp.127-135.

34. Jiménez Millán J. . “Materias Primas y Métodos de Producción de Materiales Cerámicos" , p 41-64. Eds. Sociedad Española de Arcillas.,(2001).

35. Cultrone G., de la Torre M.J., Sebastián E., Cazalla O., Rodríguez Navarro C.: "Behaviour of brickssimples in aggresive environments". Water, Air and Soil Pollution. Vol. 119, (2000), pp.191-207,

36. Jordán M.M. Sanfeliu T Hernández-Crespo M.S, Almendro-Candel, M.B., García-Sánchez E.: "Aptitudes cerámicas de una arcilla tipo modificada por la adición de un residuo rico en carbonato cálcico". Materiales Arcillosos: de la Geología a las Nuevas Aplicaciones, p. 165 Ed. M. Suárez, M. A. Vicente, V. Rives y M. J. Sánchez Salamanca, (2006)

37. Xavier E.: “Generación de residues y valoración". http:// www.cnpml.org/ html/archivos/Ponencias/Ponencias - ID56.pdf. (2004)

38. Betancourt, D., Martirena, F., Day R., Díaz Y.:"Influencia de la adición de carbonato de calcio en la eficiencia energética de la producción de ladrillos de cerámica roja". Rev. Ing. constr. Vol. 22, n³, (2007), pp. 187-196.

39. Mayoral M.C., Izquierdo M.T, Andres J.M., y Rubio B. "Aluminosilicates transformations in combustion followed by DSC". Thermochim. Acta.Vol. 373, (2001), pp. 173-180

40. Avgustinik AI.: Cerámica. Ed. Reverte. Barcelona, (1983).

41. García Ten J., Orts M.J., Saburit A., Silva G.: "Thermal conductivity of traditional ceramics. Part I: influence of bulk density and firing temperatura". Ceram. Int. Vol. 36, (2010), pp. 1951-1959.

42. García-Ten J Orts MJ Saburit A Silva G. “Thermal conductivity of traditional ceramics. Part II: influence of mineralogical composition". Ceram. Int. Vol. 36 (2010), pp. 2017-202.

Recibido: $23 / 08 / 2012$

Recibida versión corregida: 14/11/2012

Aceptado: 03/12/2012 